SLAC-TN-10-035

LCLS-TN-08-9

\title{
Undulator Transportation Test Results
}

\author{
Zachary Wolf, Nick Horton, David Kharakh, Yurii Levashov, \\ Heinz-Dieter Nuhn, Ben Poling, Ed Reese \\ SLAC
}

September 26, 2008

\begin{abstract}
A test was performed to determine whether transporting and handling the undulators makes any changes to their properties. This note documents the test. No significant changes to the test undulator were observed.
\end{abstract}

\section{Introduction $^{1}$}

After the LCLS undulators are tuned and fiducialized in the Magnetic Measurement Facility (MMF), they must be transported to storage buildings and transported to the tunnel. It has been established that the undulators are sensitive to temperature. ${ }^{2}$ We wish to know whether the undulators are also sensitive to the vibrations and shocks of transportation. To study this issue, we performed a test in which an undulator was measured in the MMF, transported to the tunnel, brought back to the MMF, and re-measured. This note documents the test and the results.

\section{Test Procedure}

The transportation test was performed on July 15, 2008. Undulator 16 (serial number) was used for the test. The undulator was placed in its crate with thermal insulating material under it. A shock sensor and two temperature sensors were placed in the crate. The shock sensor was attached to the undulator. One temperature sensor was attached to the undulator and the other measured the air temperature outside the crate. The crate was sealed. No thermal insulation was placed on top of it.

At 9:10 am, the undulator left the MMF and followed the route shown in figure 1 . The MMF is in building 81 at the left of the figure. The route ends at the undulator tunnel on the right of the figure. At the undulator tunnel, the undulator was taken off the truck, moved into the tunnel for 15 minutes, and then was brought out and put back on the truck. The undulator was not taken out of its crate. The undulator was transported along the same route back to the MMF. A forklift was used at the MMF both to load and unload the undulator from the truck. The undulator returned to the MMF at 10:40 am.

During the test, the outside temperature and the undulator temperature were recorded. The outside temperature is shown in green in figure 2. The undulator temperature is shown in red. The dotted box indicates the data of interest during the test. (The plot shows the full recorder output over two days.) After the undulator left the $68^{\circ} \mathrm{F}$ MMF, the outside temperature rose

\footnotetext{
${ }^{1}$ Work supported in part by the DOE Contract DE-AC02-76SF00515. This work was performed in support of the LCLS project at SLAC.

${ }^{2}$ Z. Wolf, Y. Levashov, E. Reese, "Undulator Changes Due To Temperature Excursions", LCLS-TN-08-8, September, 2008.
} 


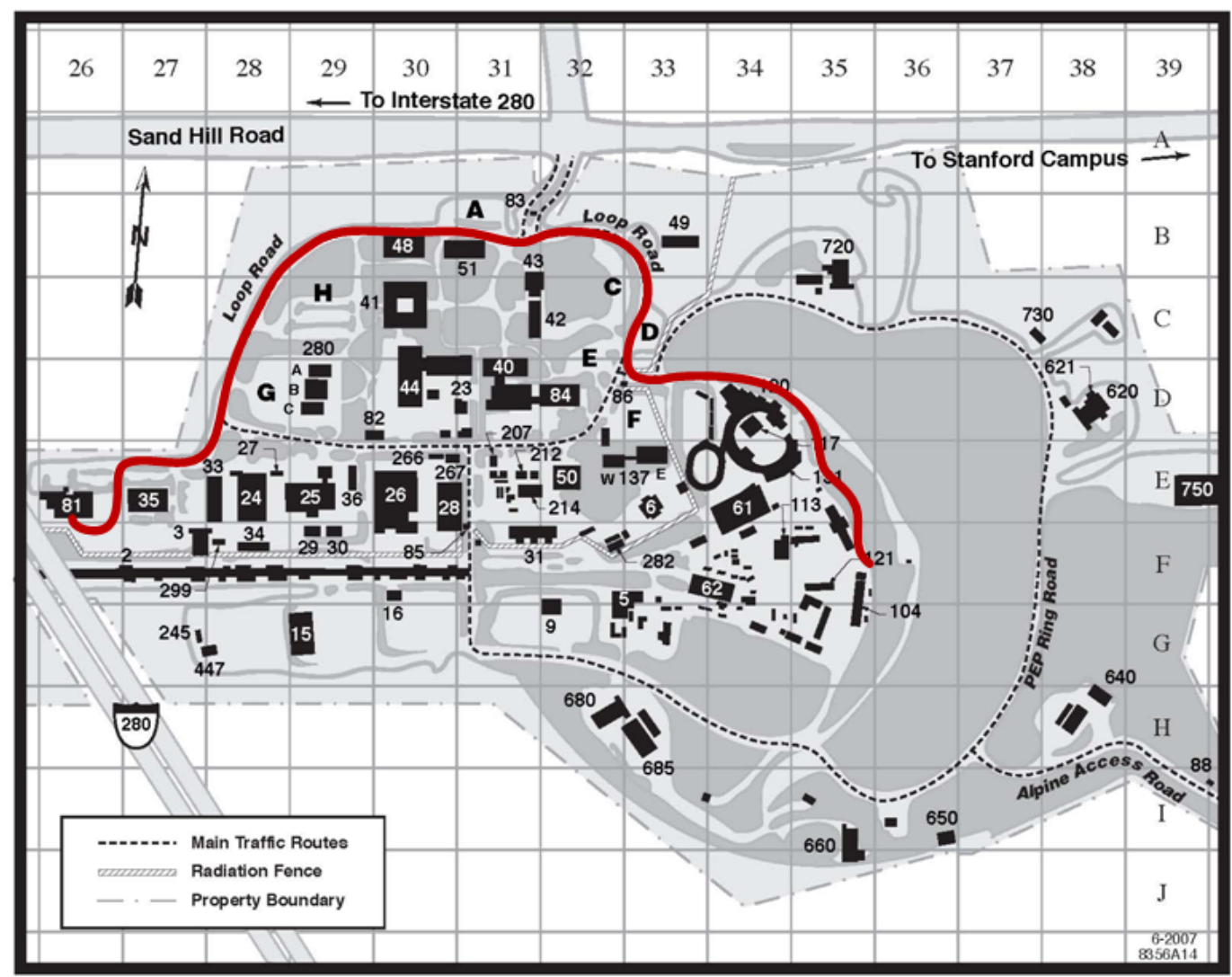

Figure 1: The red line shows the route of the transportation test.

to approximately $83^{\circ} \mathrm{F}$. The undulator temperature, however, rose only to approximately $69^{\circ} \mathrm{F}$. This is due to the thermal insulation around the undulator and large thermal mass of the undulator. This $1^{\circ} \mathrm{F}$ undulator temperature change is not expected to alter the undulator.

The undulator shock data was also recorded during the test. It consists of individual shock events in which the recorder is triggered and samples are taken. Twenty six shock events were recorded during the test. The maximum shock produced an acceleration of $0.8 \mathrm{~g}$. This event is shown in figure 3. (Note that the event number in the plot is different than the event number during the test since the recorder was turned on the day before the test.) The event consists of an impulse of short duration. Recordings of the other events are available.

\section{$3 \quad$ Test Results}

Undulator 16 was measured on 6/30/08 and the measurements are in dataset 2. The datasets are referenced in the plots which follow. After the transportation test, the undulator was re-measured on $7 / 16 / 08$ and the results are in dataset 3 .

Figure 4 shows the $\mathrm{x}$-trajectories for datasets 2 and 3 . The tolerance on the $\mathrm{x}$-trajectory is that it remain straight to $2 \mu \mathrm{m}$. (All tolerances come from a Physics Requirements Document. ${ }^{3}$ ) There is no significant change compared to the tolerance.

\footnotetext{
${ }^{3}$ H. D. Nuhn et al., "General Undulator System Requirements", LCLS Physics Requirements Document 1.4-001.
} 


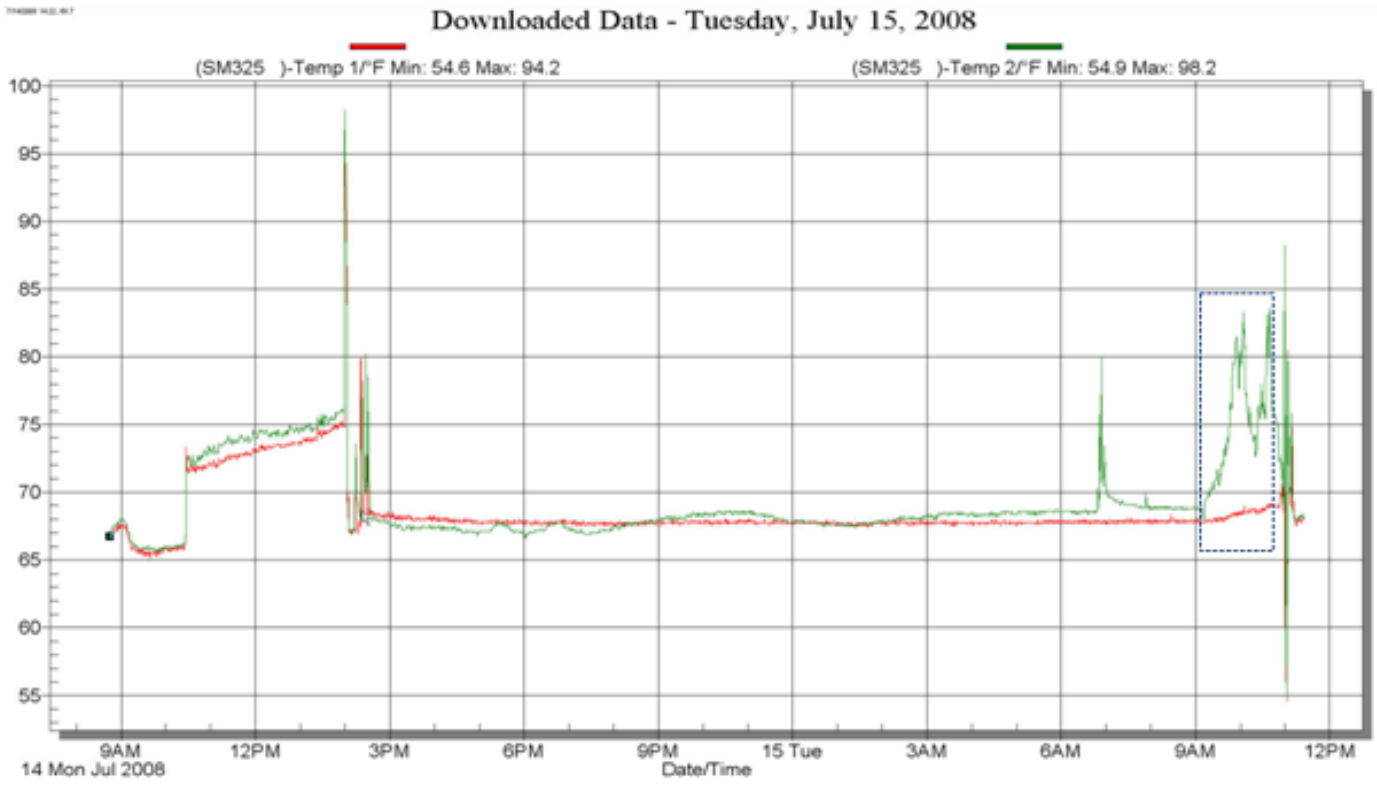

Figure 2: Undulator temperature and air temperature in the crate during the test.

Figure 5 shows the y-trajectory for datasets 2 and 3 . The tolerance on the y-trajectory is that it remain straight to $2 \mu \mathrm{m}$. Again, there is no significant change compared to the tolerance.

Figure 6 shows the change in phase between datasets 2 and 3. The phase changes are less than 1 degree. The tolerance on the rms phase error is that it be less than 10 degrees. There is no significant change. The two rows of points for the dataset 3 change is due to small slope changes in the trajectories.

The field integrals from datasets 1 and 3 are given in the table below. (Field integrals were not measured in dataset 2.) The tolerance on $I_{1 x}$ and $I_{1 y}$ is that they be below $40 \mu \mathrm{Tm}$. The tolerance on $I_{2 x}$ and $I_{2 y}$ is that they be below $50 \mu \mathrm{Tm}^{2}$. The field integrals stayed well within tolerance.

\begin{tabular}{|r|r|r|}
\hline & Dataset 1 & Dataset 3 \\
\hline$I_{1 x}(\mu \mathrm{Tm})$ & -9.0 & 7.0 \\
\hline$I_{2 x}\left(\mu \mathrm{Tm}^{2}\right)$ & -21.4 & -32.6 \\
\hline$I_{1 y}(\mu \mathrm{Tm})$ & -2.0 & -8.3 \\
\hline$I_{2 y}\left(\mu \mathrm{Tm}^{2}\right)$ & 2.4 & 3.2 \\
\hline
\end{tabular}

Field integrals before and after the test.

The phase errors from datasets 2 and 3 are given in the following table. The tolerance on all the phase errors in the table is $10 \mathrm{deg}$. All phase errors stayed well within tolerance.

\begin{tabular}{|r|r|r|}
\hline & Dataset 2 & Dataset 3 \\
\hline rms phase error (deg) & 3.6 & 3.6 \\
\hline phase matching error at entrance $(\mathrm{deg})$ & 0.8 & 0.9 \\
\hline phase matching error at exit $(\mathrm{deg})$ & 2.0 & 2.1 \\
\hline phase error of cell $(\mathrm{deg})$ & 2.6 & 3.1 \\
\hline
\end{tabular}

Phase errors before and after the test. 


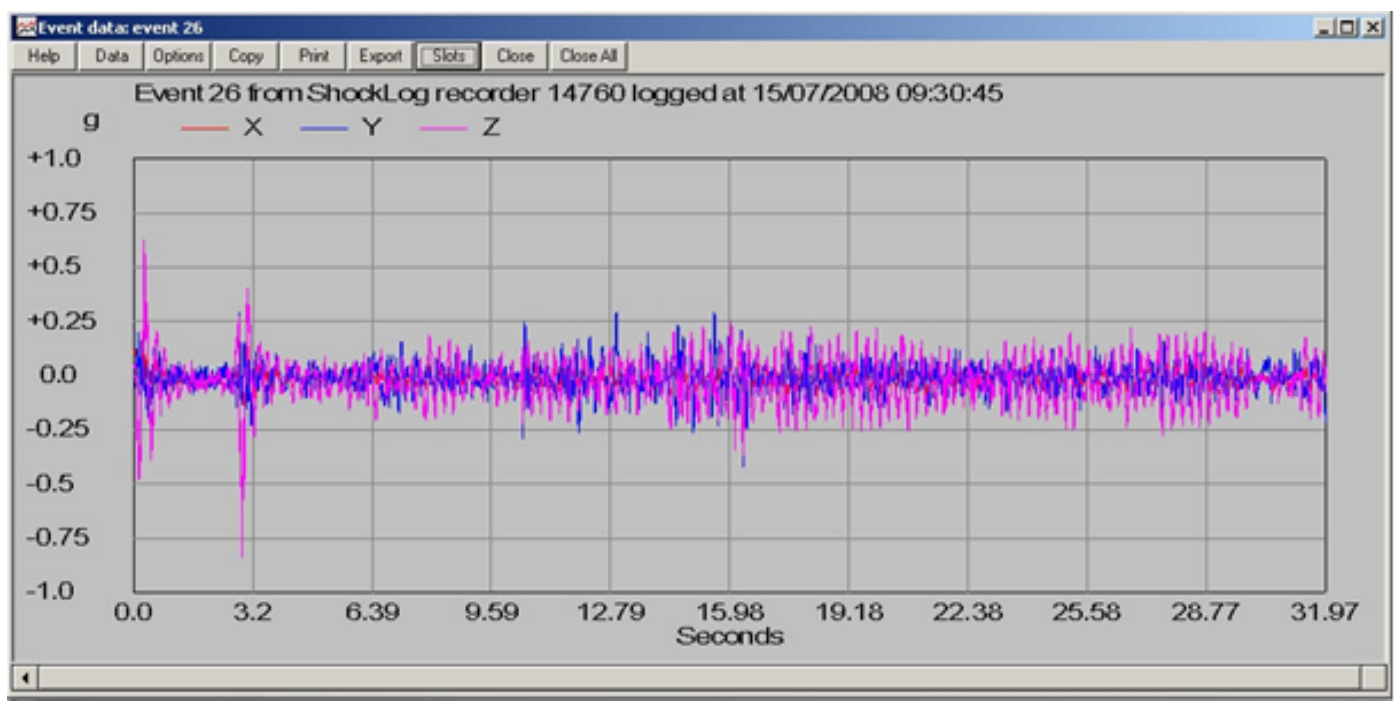

Figure 3: The shock data consists of 26 recorded events. This event produced the largest acceleration.

The undulators are fiducialized by giving the position of the beam axis relative to tooling balls. The fiducialization information for datasets 2 and 3 are given in the table below. The table shows the average of the four horizontal and four vertical measurements of the beam axis position relative to the tooling balls on the undulator. The beam axis position changes are far below the fiducialization accuracy requirements of $50 \mu \mathrm{m}$ in $\mathrm{x}$, and $40 \mu \mathrm{m}$ in $\mathrm{y}$.

\begin{tabular}{|l|r|r|r|}
\hline & Dataset 2 & Dataset 3 & Change \\
\hline X Position $(\mathrm{mm})$ & 248.625 & 248.638 & 0.013 \\
\hline Y Position $(\mathrm{mm})$ & 179.742 & 179.745 & 0.003 \\
\hline
\end{tabular}

Fiducialization data before and after the test.

\section{Conclusion}

No significant changes occurred to the undulator during the transportation test. It is safe to transport the undulators under the conditions of this test. 


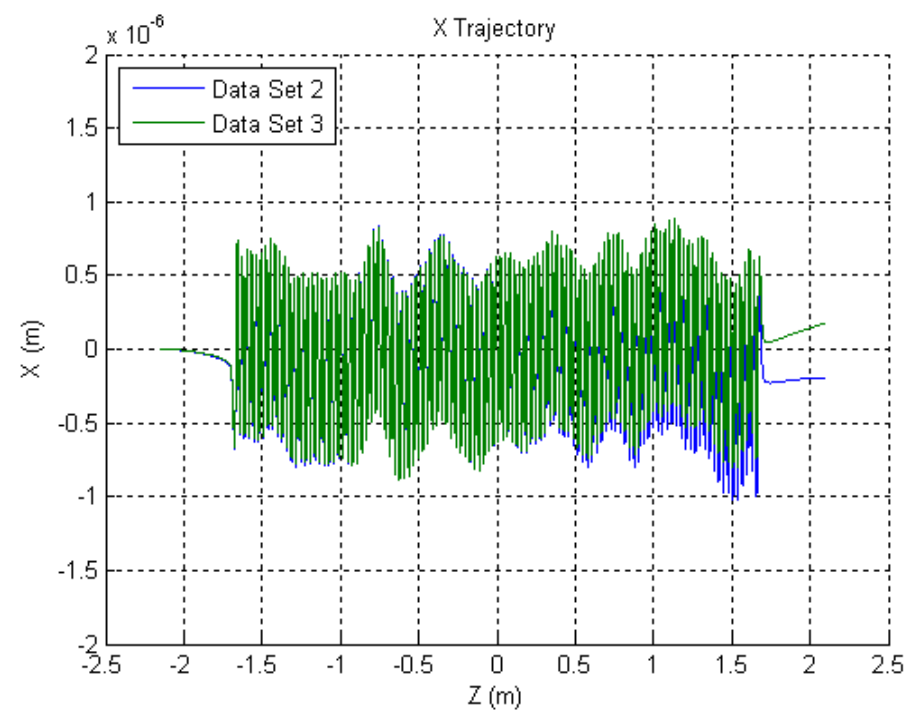

Figure 4: X-trajectory for datasets 2 and 3.

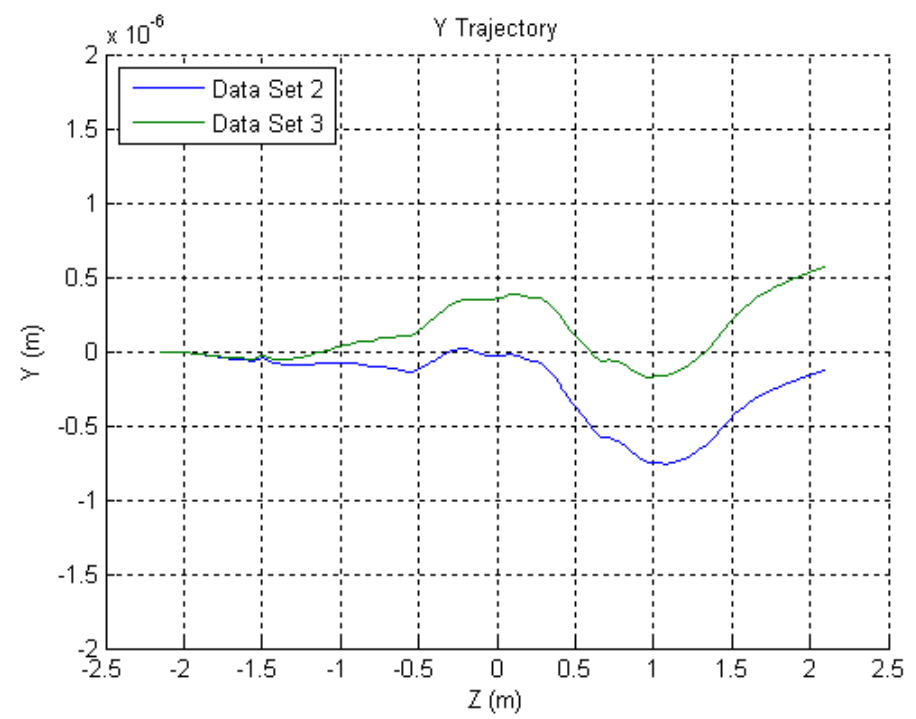

Figure 5: Y-trajectory for datasets 2 and 3. 


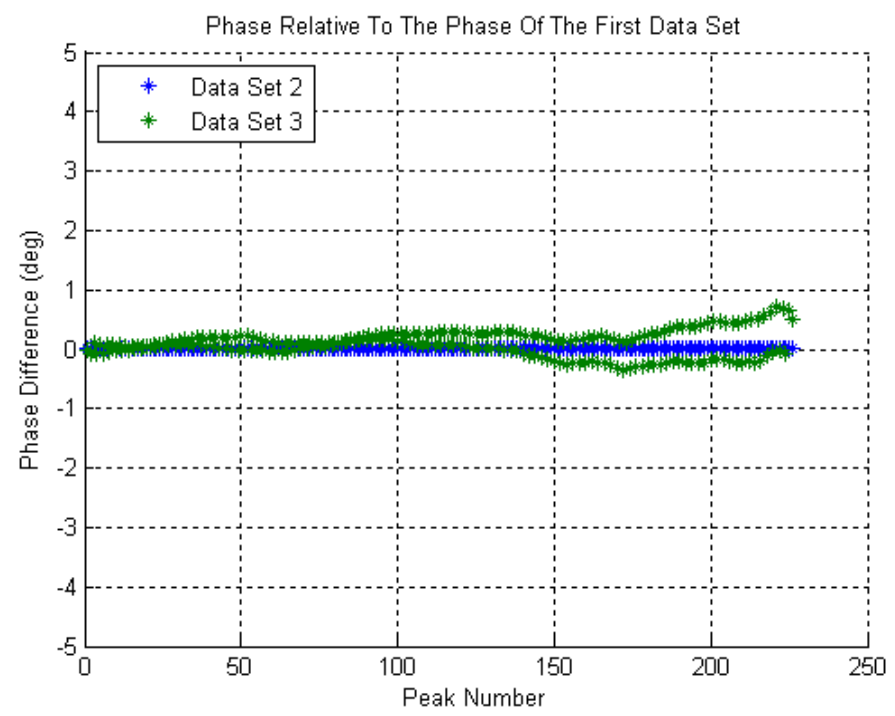

Figure 6: Change in phase between datasets 2 and 3. 